\title{
(3R)-5,6,7-trihydroxy-3-isopropyl-3-methylisochroman-1-one inhibited osteosarcoma growth by inducing apoptosis
}

\author{
MING-ZHU SONG ${ }^{1 *}$, FENG-LIN ZHANG ${ }^{2 *}$ and LE-JUN LIN $^{3}$ \\ ${ }^{1}$ Department of Orthopaedics and Traumatology, Yantaishan Hospital; ${ }^{2}$ Department of Emergency, \\ Yantai Hospital of Traditional Chinese Medicine; ${ }^{3}$ Department of Nuclear Medicine, The Affiliated Yantai \\ Yuhuangding Hospital of Qingdao University Medical College, Yantai, Shandong 264000, P.R. China
}

Received September 16, 2018; Accepted May 9, 2019

DOI: $10.3892 /$ etm.2019.7681

\begin{abstract}
As one of the leading causes of cancer-associated mortalities worldwide, the overall survival rate of osteosarcoma has stably remained at $15-30 \%$ for several decades. (3R)5,6,7-trihydroxy-3-isopropyl-3-methylisochroman-1-one (TIM), isolated from the whole plant of Selaginella moellendorffii Hieron., has been reported to have pharmacological activities. In the present study, the anti-proliferative effects of TIM against osteosarcoma were evaluated, and the underlying molecular mechanisms were explored. The results demonstrated that TIM inhibited proliferation and induced apoptosis in U2OS cells. Furthermore, the expression of the pro-apoptotic protein NOXA in the intrinsic apoptosis pathway was upregulated by TIM, while the expression of myeloid cell leukemia 1 , an anti-apoptotic protein, was downregulated. In addition, TIM increased the protein expression of the endoplasmic reticulum stress markers inositol-requiring enzyme 1, activating transcription factor 6 and glucose-regulated protein 78. These results suggested that TIM induced ER stress response while activating intrinsic apoptosis. Furthermore, treating osteosarcoma tumor-bearing mice with TIM significantly inhibited the tumor growth in the xenograft animal model. Overall, the study results suggested that TIM may serve as a potential antitumor agent against osteosarcoma.
\end{abstract}

\section{Introduction}

Osteosarcoma is the third most common cancer in children and young adults, and the most common primary malignant

Correspondence to: Dr Le-Jun Lin, Department of Nuclear Medicine, The Affiliated Yantai Yuhuangding Hospital of Qingdao University Medical College, 20 Yuhuangding East Road, Yantai, Shandong 264000, P.R. China

E-mail: starplace2020@yeah.net

${ }^{*}$ Contributed equally

Key words: (3R)-5,6,7-trihydroxy-3-isopropyl-3-methylisochroman1-one, osteosarcoma, apoptosis, endoplasmic reticulum stress bone tumor in the pediatric and adult populations. It is characterized by the proliferation of tumor cells producing osteoid or immature bone matrix $(1,2)$. Approximately $15-20 \%$ of osteosarcoma patients present with pulmonary metastasis at the time of diagnosis, exhibiting an extremely poor 5-year survival rate. Despite the availability of multimodality treatment, numerous challenges remain in the clinical treatment of osteosarcoma patients, and the majority of chemotherapeutic drugs have a limited effect on osteosarcoma due to side effects and development of drug resistance. The poor prognosis of osteosarcoma patients has not improved over the three decades following the advent of modern chemotherapy (3-5). Therefore, there is an urgent need to develop novel therapeutic agents against osteosarcoma.

Apoptosis, regulated by complex signaling pathways, is essential for normal tissue development (6). The abnormal expression of pro- or anti-apoptotic genes is believed to be associated with a number of pathologic disorders, including cancer $(7,8)$. As a major signaling cascade for apoptosis, the intrinsic mitochondrial pathway serves crucial roles in the regulation of apoptotic processes in various cancer cells (9). Studies have demonstrated that persistent endoplasmic reticulum (ER) stress was associated with the activation of the intrinsic mitochondrial apoptosis pathway (10). ER stress is activated through three sensors: Glucose-regulated protein 78 (GRP78), inositol-requiring enzyme 1 (IRE1) and activating transcription factor 6 (ATF6) (11). Once the sensors are released from GRP78 (also known as binding immunoglobulin protein), certain downstream effectors, such as C/EBP homologous protein, are activated to trigger pro-apoptotic signals by targeting several apoptotic genes $(12,13)$.

Natural products isolated from plants have recently attracted great interest due to their potent biological and pharmaceutical activities. A novel compound known as $(3 R)$ 5,6,7-trihydroxy-3-isopropyl-3-methylisochroman-1-one (TIM), isolated from the whole plants of Selaginella moellendorffii Hieron., has been reported to inhibit the proliferation of colon cancer cells by inducing apoptosis (14). In the present study, TIM was further studied to investigate its effects on osteosarcoma. The study results revealed that TIM exhibited potent antitumor activities, as evidenced by its inhibitory effect on osteosarcoma growth in a xenograft tumor model. These results provided 
insights that may facilitate the development of TIM as a new potential therapeutic agent against osteosarcoma.

\section{Materials and methods}

Cell lines, reagents and chemicals. The normal osteoblast cell line MC3T3-E1, breast adenocarcinoma cell line MCF-7, pancreatic adenocarcinoma cell line PANC-1, colon adenocarcinoma cell line HT-29, stomach adenocarcinoma cell line BGC-823, lung adenocarcinoma cell line A549, hepatoblastoma cell line HepG2, glioblastoma cell line U-251 MG, epidermoid carcinoma cell line A431, and osteosarcoma cell lines U2OS, MG63, 143B and SaOS-2 were purchased from Shanghai Cell Bank (Shanghai, China). Cell culture media and fetal bovine serum (FBS) were purchased from Gibco (Thermo Fisher Scientific, Inc., Waltham, MA, USA). The fluorescent probe 5,5',6,6'-tetrachloro-1,1',3,3'-tetraethylbenzimidazolylcarbocyanine iodide (JC-1) was purchased from Molecular Probes (Eugene, OR, USA). The 3-(4,5-dimethylthiazol-2-yl)-2,5-diphenyltetrazolium-bromide (MTT) was purchased from Sigma-Aldrich (Merck KGaA, Darmstadt, Germany). The Annexin V-FITC/propidium iodide (PI) apoptosis detection kit was purchased from BIO-BOX Biotech (Nanjing, China), while the cytochrome $c$ immunoassay kit was from R\&D Systems, Inc. (Minneapolis, MN, USA). iScript ${ }^{\mathrm{TM}}$ Reverse Transcription Supermix for RT-qPCR (cat. no. 170-88400) was purchased from Bio-Rad Laboratories, Inc. (Hercules, CA, USA). SYBR ${ }^{\mathrm{TM}}$ Green Master Mix kit for PCR (cat. no. 4309155) was purchased from Thermo Fisher Scientific, Inc. Doxorubicin and Paclitaxel were purchased from Shijiazhuang Pharmaceutical Co., Ltd. (Shijiazhuang, China), and prostaglandin E1 $\left(\mathrm{PGE}_{1}\right)$ was from Merck KGaA (Calbiochem; La Jolla, CA, USA). All other solvents were of analytical grade and were obtained from Sinopharm Chemical Reagent Co., Ltd. (Shanghai, China). TIM was isolated and identified by Professor Yi He from the Department of Pharmaceutical Science, Hangzhou Vocational and Technical College (Hangzhou, China) for use in the present study (14). TIM was dissolved in DMSO for use in the in vitro experiments. For the in vivo experiments, doxorubicin was freshly prepared with PBS and TIM was freshly prepared in $0.5 \%$ carboxymethyl cellulose prior to use in the in vivo experiments.

Animals. Female nude mice (BALB/c-nu/nu; age, 6 weeks) were obtained from Vital River Laboratory Animal Technology Co., Ltd. (Beijing, China), and housed in a sterile environment at $25^{\circ} \mathrm{C}$ on a 12 -h light/dark cycle. The mice had free access to food and water during the entire experimental period. All animal procedures were conducted in accordance with the Chinese Legislation Guide for the Care and Use of Laboratory Animals, and the study was approved by the Institutional Animal Care and Use Committee of Qingdao University Medical College (Yantai, China).

Cell culture, treatment and viability assay. MC3T3-E1, MCF-7, PANC-1, HepG2, A431, MG63 and 143B cells were cultured in Dulbecco's modified Eagle's medium, and HT-29, BGC-823, A549, U-251 MG, U2OS and SaOS-2 cells were cultured in RPMI 1640 medium. Both media were supplemented with $10 \% \mathrm{FBS}$ and $100 \mu \mathrm{g} / \mathrm{ml}$ penicillin/streptomycin at $37^{\circ} \mathrm{C}$ in an atmosphere containing $5 \% \mathrm{CO}_{2}$. For the $\mathrm{IC}_{50}$ assay, cells were treated with Paclitaxel $(1,5,25,125,625,3,125$ or $5,000 \mathrm{nM})$ or $\operatorname{TIM}(1,5,25,125,625,3,125$ or $5,000 \mathrm{nM})$ alone for $48 \mathrm{~h}$. Following the $\mathrm{IC}_{50}$ assay, U2OS cells were treated with TIM $(4 \mathrm{nM})$ together with $\mathrm{PGE}_{1}(100 \mathrm{nM})$ for $48 \mathrm{~h}$. Cells treated with DMSO were used as control. Subsequently, the cell viability was measured by an MTT assay. Briefly, treated cells were seeded into 96 -well plates at a density of $4 \times 10^{4} / 100 \mu \mathrm{l} /$ well. MTT solution $(10 \mu \mathrm{l})$ was added to each well and incubated at $37^{\circ} \mathrm{C}$ for $4 \mathrm{~h}$. Then $200 \mu \mathrm{l}$ of DMSO was added into each well to dissolve the formazan. The absorbance was measured at $570 \mathrm{~nm}$. The $\mathrm{IC}_{50}$ value was calculated with GraphPad Prism software (version 8; GraphPad Software, Inc.).

For subsequent experiments except for the xenograft tumor model establishment and terminal deoxynucleotidyl transferase dUTP nick end labeling (TUNEL) assay, U2OS cells were treated with TIM $(1,2$ or $4 \mathrm{nM})$ for $48 \mathrm{~h}$. Cells treated with DMSO were used as control.

Cytochrome c assay. U2OS cells were fractionated with Fractionation kit (cat. no. 78840; Thermo Fisher Scientific, Inc.) subsequent to TIM treatment. The cytochrome $c$ release was measured using a cytochrome c quantikine ELISA kit (cat. no. DCTC0; R\&D Systems, Inc.), according to the manufacturer's protocol.

Mitochondrial membrane potential (MMP) measurement. MMP was measured using the fluorescent probe JC-1. Briefly, following the indicated treatments, U2OS cells were incubated with JC-1 for $15 \mathrm{~min}$ at $37^{\circ} \mathrm{C}$ in the dark. Subsequent to rinsing twice with PBS, the red/green fluorescence intensity was determined with a fluorescence microplate reader (Tecan Polarion; Tecan Group, Ltd., Mannedorf, Switzerland) at an excitation wavelength of $490 \mathrm{~nm}$ and emission wavelength of $530 / 590 \mathrm{~nm}$, respectively.

Caspase activity measurement. Following the indicated treatments, U2OS cells were lysed with the fractionation kit. Next, the caspase-3/9 activities were determined using the assay kits (cat. no. K106-100 for caspase-3; cat. no. K119-110 for caspase-9; R\&D Systems, Inc.) according to the manufacturer's protocol, followed by examination with a microplate reader.

DNA fragmentation measurement. Subsequent to the indicated treatments, U2OS cells were lysed with the fractionation kit, and DNA fragmentation was measured by the Cell Death Detection ELISA ${ }^{\text {plus }}$ kit (cat. no. 11544675001; Roche Diagnostics) according to the manufacturer's protocol. The absorbance was measured with a microplate reader at $405 \mathrm{~nm}$.

Cell cycle distribution measurement. Subsequent to the indicated treatments, U2OS cells were washed three times with PBS and fixed with $70 \%$ ethanol for 30 min at $4^{\circ} \mathrm{C}$. Next, the cells were incubated with PI/Triton X-100 for $10 \mathrm{~min}$, and the cell cycle distribution was examined by flow cytometry (BD Biosciences, San Jose, CA, USA). The ratio of G1/S was calculated, and changes in cell cycle distribution were expressed as a percentage of the control, which was set to $100 \%$.

Apoptosis analysis. For apoptosis analysis, a total of $2 \times 10^{6} \mathrm{U} 2 \mathrm{OS}$ cells were harvested, washed with pre-chilled PBS, and then re-suspended in $500 \mu \mathrm{l}$ binding buffer. Subsequently, $5 \mu \mathrm{l}$ Annexin 
V-FITC/PI were added to each sample, and incubated for $10 \mathrm{~min}$ in the dark at room temperature. Analysis of apoptotic cells was performed using a FACScan flow cytometer (BD Biosciences).

$R T-q P C R$. Total RNA was extracted with the Qiagen RNeasy reagents (Qiagen $\mathrm{GmbH}$, Hilden, Germany) following manufacturer's protocol and the RNA concentration was measured with NanoDrop Spectrophotometer. RNA $(0.5 \mu \mathrm{g})$ was used to generate cDNA using an iScript ${ }^{\mathrm{TM}}$ Reverse Transcription Supermix kit; the RT protocol was as follows: $25^{\circ} \mathrm{C}$ for $5 \mathrm{~min}, 46^{\circ} \mathrm{C}$ for $20 \mathrm{~min}$ and $95^{\circ} \mathrm{C}$ for $1 \mathrm{~min}$. qPCR was run on a StepOne system (Thermo Fisher Scientific, Inc.) using SYBR Green Supermix (Thermo Fisher Scientific, Inc.). The thermocycling conditions were as follows: $95^{\circ} \mathrm{C}$ for $10 \mathrm{~min}$, and 40 cycles of $95^{\circ} \mathrm{C}$ for $15 \mathrm{sec}$ and $60^{\circ} \mathrm{C}$ for $60 \mathrm{sec}$. The mRNA levels were quantified with the comparative $\mathrm{C}_{\mathrm{q}}$ value method (15) and normalized to $\beta$-actin levels. The gene-specific primer sequences were as follows: Caspase-3 forward, 5'-ttgtggaattgatgcgtgat-3' and reverse, 3'-ggcaggcct gaataatgaaa-5' (GenBank reference: AJ413269.1); Caspase-9 forward, 5'-caggagaaaggcctcagttg-3' and reverse, 3'-ggatgtagc cgtgtgacctt-5' (GenBank reference: AY214168.1); Irel forward, 5'-cggectttgcagatagtctc-3' and reverse, 3'-actgtccacagtcaccac ca-5' (GenBank reference: AF059198.1); Atf6 forward, 5'-tga acttcgaggatgggttc-3' and reverse, 3'-tcactccctgagttcctgct-5 (GenBank reference: AB015856.1); Grp78 forward, 5'-aaa ggacaggctggtgctaa-3' and reverse, 3'-gggctggagtacagtggtgt-5' (GenBank reference: BC020235.1); $\beta$-actin forward, 5'-aga gctacgagctgcetgac-3' and reverse, 3'-agcactgtgttggcgtacag-5 (GenBank reference: AK222925.1).

Western blot analysis. In order to obtain the total protein, cells were lysed with th fractionation kit and centrifuged at $6,000 \mathrm{x} \mathrm{g}$ at $4^{\circ} \mathrm{C}$ for $15 \mathrm{~min}$, following which protein concentration was determined by the BCA method. Next, $40 \mu \mathrm{g}$ protein samples were subjected to sodium dodecyl sulfate-polyacrylamide gel electrophoresis with $4-12 \%$ gels and then transferred to polyvinylidene difluoride membranes. The membranes were blocked with $1 \%$ bovine serum albumin (cat. no. A9306; Sigma-Aldrich; Merck KGaA) for $1 \mathrm{~h}$ at room temperature and then incubated at $4{ }^{\circ} \mathrm{C}$ overnight with the following primary antibodies (Abs): Anti-cleaved caspase-3 rabbit monoclonal (m)Ab (1:1,000; cat. no. 9664; Cell Signaling Technology, Inc.); anti-cleaved caspase- 9 rabbit polyclonal (p)Ab (1:500; cat. no. C7729; Sigma-Aldrich; Merck KGaA); anti-Bcl-2 rabbit mAb (1:1,000; cat. no. ab32124; Abcam); anti-Bax rabbit mAb (1:1,000; cat. no. ab182733; Abcam); anti-myeloid cell leukemia 1 (MCL-1) rabbit mAb $(1: 2,000$; cat. no. ab32087; Abcam); anti-NOXA rabbit pAb (1:500; cat. no. ab36833; Abcam); anti-IRE1 (phosphor S724) rabbit pAb (1:1,000; cat. no. ab48187; Abcam); anti-ATF6 rabbit pAb (1:500; cat. no. ab37149; Abcam); anti-GRP78 rabbit mAb (1:5,000; cat. no. ab108615; Abcam); anti- $\beta$-actin rabbit $\mathrm{mAb}$ (1:5,000; cat. no. ab179467; Abcam). Membranes were then incubated with horseradish peroxidase-conjugated goat anti-rabbit secondary antibodies (1:8,000; cat. no. ab6721; Abcam) for $1 \mathrm{~h}$ at room temperature. The bands were then detected with an ECL system (EMD Millipore, Billerica, MA, USA). The bands were quantified with ImageJ software (version 1.4; National Institutes of Health, Bethesda, MD, USA).
Xenograft tumor model. A total of $5 \times 10^{6} \mathrm{U} 2 \mathrm{OS}$ cells were injected subcutaneously into the right flank of nude mice. When the tumor volume reached $50-100 \mathrm{~mm}^{3}$, the tumor-bearing mice were divided into four groups ( 5 mice per group), and administered different treatments. One of the groups was intraperitoneally injected with doxorubicin $(2 \mathrm{mg} / \mathrm{kg})$ twice weekly, while two other mouse groups received intragastric treatment with TIM (1 and $2 \mathrm{mg} / \mathrm{kg}$ ), 5 days per week. Mice treated with PBS served as the negative controls. Once the tumor volume reached $1,500 \mathrm{~mm}^{3}$ (after $\sim 3$ weeks), all tumor-bearing mice were sacrificed to collect the tumor tissue to record the tumor weight and detect apoptosis.

TUNEL assay. Tumor samples were fixed with $4 \%$ paraformaldehyde for $4 \mathrm{~h}$, and then dehydrated by graded sucrose solution. Subsequently, the tumor samples were embedded in Tissue-Tek Optimal Cutting Temperature compound (Sakura, Alphen aan den Rijn, The Netherlands), and $10-\mu \mathrm{m}$ frozen sections were cut sagittally with a freezing microtome. Apoptosis was detected by the In Situ Cell Death Detection kit (Roche Applied Science, IN, USA). Briefly, frozen sections were incubated with permeabilization solution for $2 \mathrm{~min}$ on ice. After washing, the sections were incubated with the TUNEL reaction mixture for $60 \mathrm{~min}$ at $37^{\circ} \mathrm{C}$ in the dark and then rinsed with PBS. Subsequently, the sections were sealed with VECTASHIELD mounting medium with DAPI (Vector Laboratories, Inc., Burlingame, CA, USA) and visualized under the Olympus BX60 microscope (Olympus Corporation, Shinjuku, Japan).

Statistical analysis. Data are expressed as the mean \pm standard deviation, and the software SPSS (version 12.0; SPSS, Inc., Chicago, IL, USA) was used for statistical analysis. Data were analyzed by one-way analysis of variance, followed by the least significant difference test. A P-value of $<0.05$ was considered as an indicator of a statistically significant difference.

\section{Results}

TIM inhibits cell proliferation. To explore the anti-tumor spectrum of TIM, MTT assay was used to determine the anti-proliferative activities of TIM on different cancer cell lines. Lower $\mathrm{IC}_{50}$ values indicated stronger anti-proliferative activities. As shown in Table I, TIM selectively inhibited the proliferation of colon carcinoma and osteosarcoma cells. Furthermore, TIM exhibited low toxicity on the normal osteoblast cells MC3T3-E1 (Table I).

TIM induces the apoptosis of U2OS cells. U2OS osteosarcoma cells were subjected to TIM treatment at concentrations of 1, 2 and $4 \mathrm{nM}$, and then a number of apoptosis-associated markers were detected. Comparing with the control group, TIM treatment at concentrations of 2 and $4 \mathrm{nM}$ significantly decreased the MMP (Fig. 1A), while it markedly increased the cytochrome $c$ release (Fig. 1B), DNA fragmentation (Fig. 1C) and Caspase-3/9 activities (Fig. 1D). In addition, TIM treatment ( 2 and $4 \mathrm{nM}$ ) induced apoptosis in U2OS cells (Fig. 1E and F). However, as shown in Fig. 2, TIM treatment did not significantly alter the cell cycle distribution. Comparing with the control group, pro-apoptotic gene (Caspase-3 and Caspase-9) expression levels were significantly increased following $4 \mathrm{nM}$ 
Table I. Anti-proliferative effects of TIM on various cancer cells.

\begin{tabular}{llrr}
\hline Cell line & \multicolumn{1}{c}{ Origin } & TIM $\left(\mathrm{IC}_{50} ; \mathrm{nM}\right)$ & Paclitaxel $(\mathrm{IC} 50 ; \mathrm{nM})$ \\
\hline MC3T3-E1 & Normal osteoblast cell line & $>5,000$ & $>5,000$ \\
MCF-7 & Breast adenocarcinoma & $2,561.5 \pm 189.3$ & $6.1 \pm 1.2$ \\
PANC-1 & Pancreatic adenocarcinoma & $155.7 \pm 14.3$ & $15.3 \pm 1.7$ \\
U2OS & Osteosarcoma & $8.1 \pm 1.5$ & $16.7 \pm 2.9$ \\
MG63 & Osteosarcoma & $9.3 \pm 1.8$ & $12.5 \pm 2.2$ \\
143B & Osteosarcoma & $11.5 \pm 1.9$ & $19.2 \pm 3.5$ \\
SaOS-2 & Osteosarcoma & $15.4 \pm 2.6$ & $11.3 \pm 2.1$ \\
HT-29 & Colon adenocarcinoma & $9.5 \pm 1.7$ & $11.2 \pm 3.0$ \\
BGC-823 & Stomach adenocarcinoma & $1,332.4 \pm 127.6$ & $16.6 \pm 2.3$ \\
A549 & Lung adenocarcinoma & $44.5 \pm 5.1$ & $9.9 \pm 1.8$ \\
HepG2 & Hepatoblastoma & $1,851.5 \pm 163.2$ & $35.5 \pm 3.3$ \\
U-251 MG & Glioblastoma & $1,305.1 \pm 112.3$ & $11.3 \pm 2.2$ \\
A431 & Epidermoid carcinoma & $19.3 \pm 2.8$ & $17.5 \pm 1.9$
\end{tabular}

TIM, (3R)-5,6,7-trihydroxy-3-isopropyl-3-methylisochroman-1-one; $\mathrm{IC}_{50}$, half maximal inhibitory concentration.

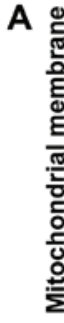
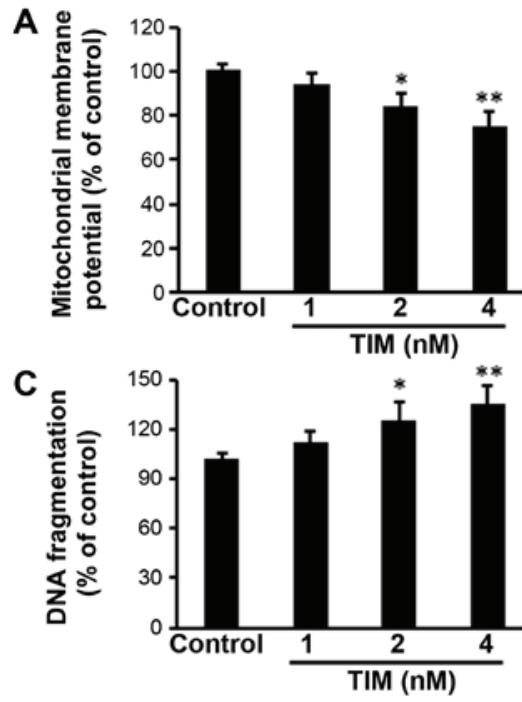

E
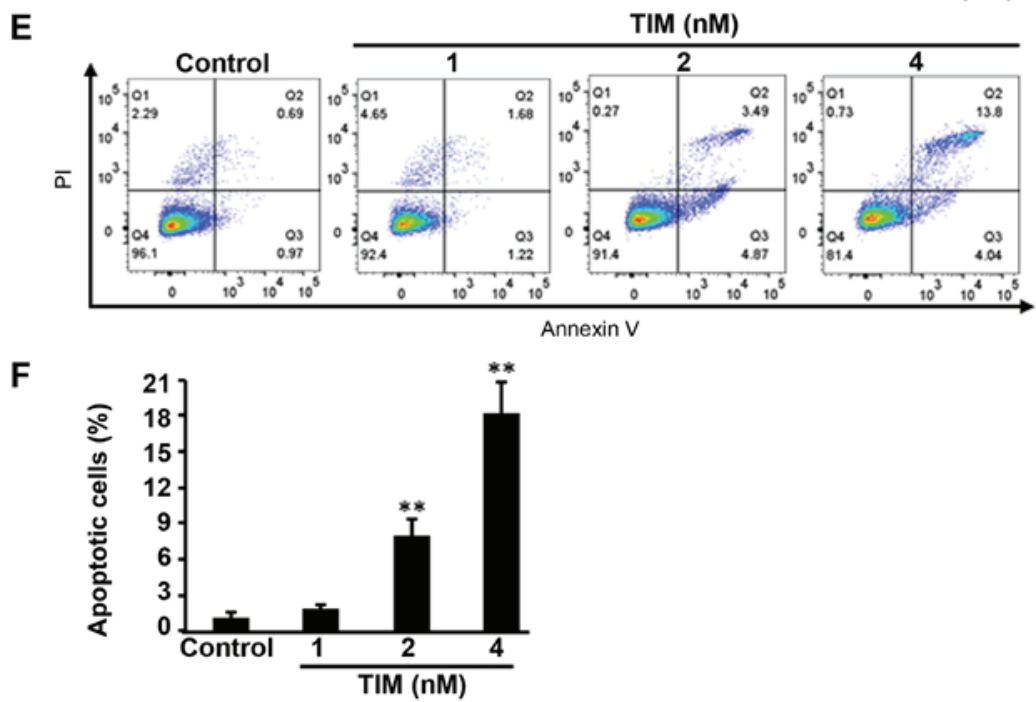
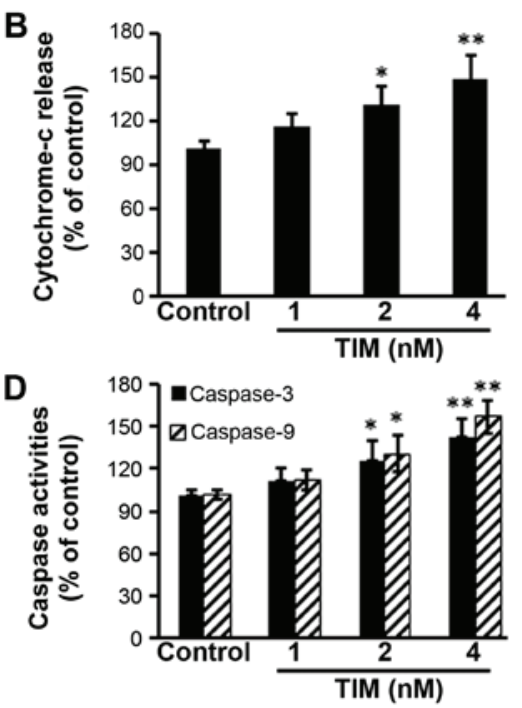

TIM (nM)
$6.1 \pm 1.2$

$5.3 \pm 1.7$

$16.7 \pm 2.9$

$12.5 \pm 2.2$

$19.2 \pm 3.5$

$11.3 \pm 2.1$

$11.2 \pm 3.0$

$9.9 \pm 1.8$

$35.5 \pm 3.3$

$17.5 \pm 1.9$ 

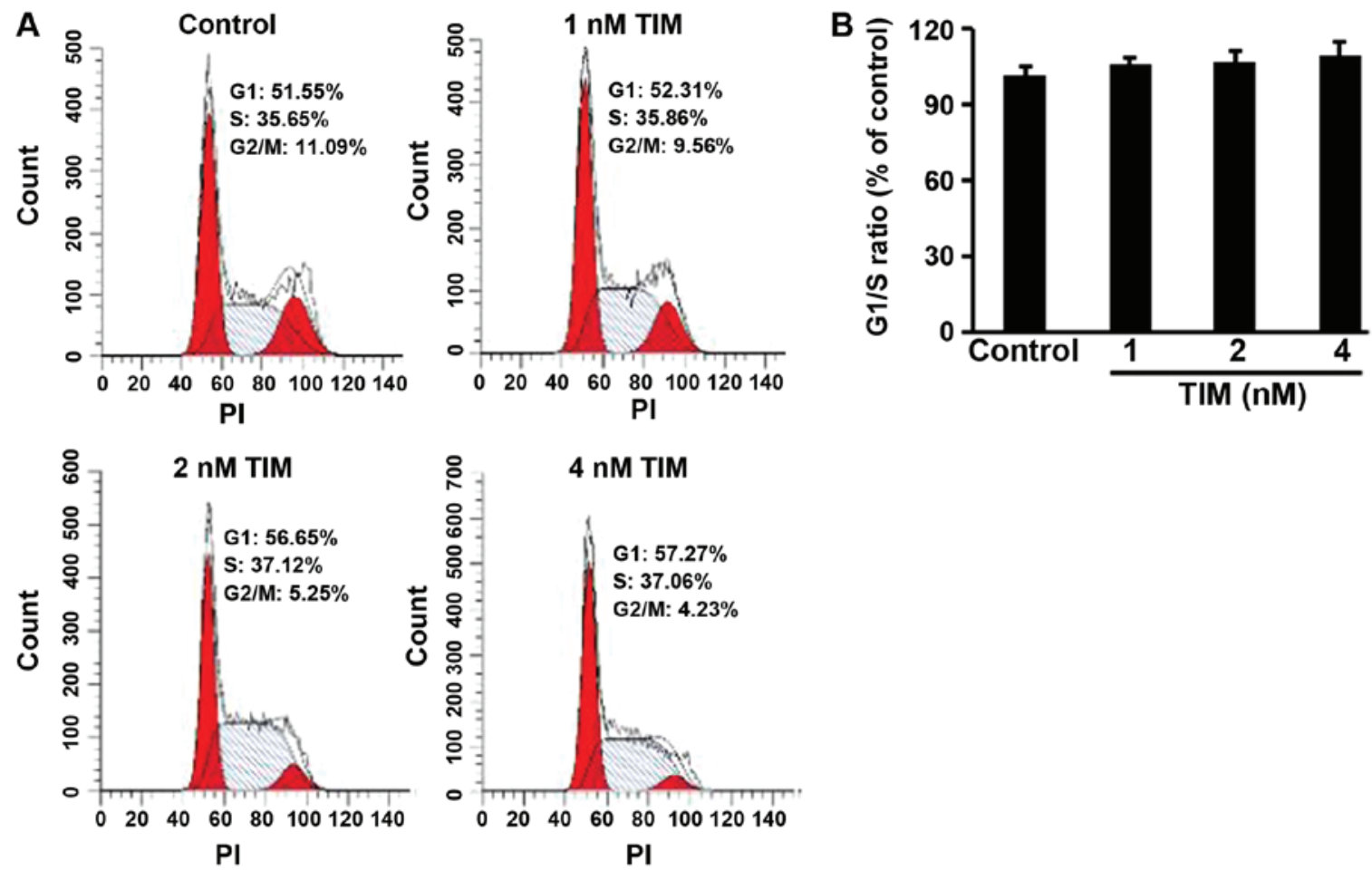

Figure 2. TIM did not alter the cell cycle distribution of U2OS cells. (A) Representative images of the flow cytometry plots for the cell cycle analysis. (B) TIM did not significantly alter the proportion of cells at G1/S phase, as compared with that in the control group. Data are expressed as the mean \pm standard deviation (n=3). TIM, (3R)-5,6,7-trihydroxy-3-isopropyl-3-methylisochroman-1-one; PI, propidium iodide.

A

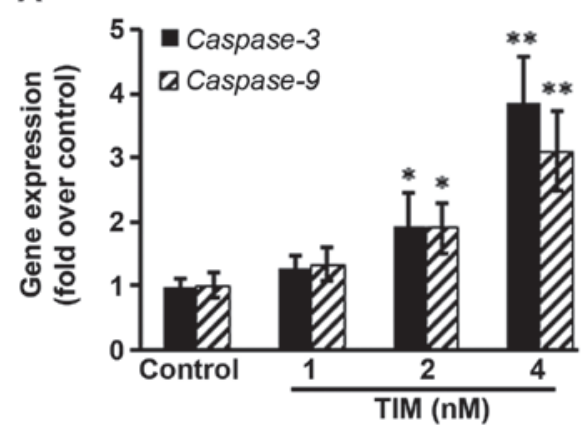

B

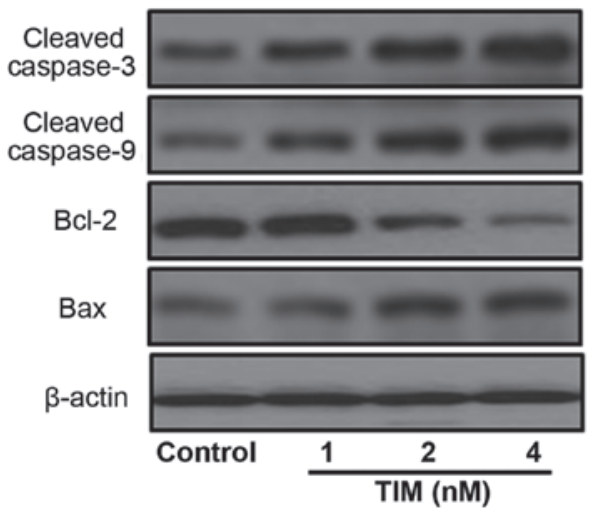

Figure 3. TIM changed the expression levels of apoptosis-associated genes and proteins in U2OS cells following treatment for $48 \mathrm{~h}$. (A) TIM increased caspase gene expression. (B) TIM treatment increased the expression levels of pro-apoptotic proteins, and decreased the expression levels of anti-apoptotic proteins. Data are expressed as the mean \pm standard deviation $(\mathrm{n}=3)$. ${ }^{*} \mathrm{P}<0.05$ and ${ }^{* *} \mathrm{P}<0.01$, vs. control group. TIM, (3R)-5,6,7-trihydroxy-3-isopropyl-3-methylisochroman-1-one; Bcl-2, B-cell lymphoma 2; Bax, Bcl-2-associated X protein.

TIM treatment. Pro-apoptotic protein (Cleaved caspase-3, Cleaved caspase-9 and Bax) expression levels were markedly increased following TIM treatment, whereas anti-apoptotic protein (Bcl-2) expression was evidently decreased (Fig. 3).

TIM induces apoptosis through the NOXA/MCL-1 axis and by triggering ER stress. The NOXA/MCL-1 axis is involved in chemotherapeutic-induced apoptosis in several tumor types (16). The present study results demonstrated that the protein expression of MCL-1 was decreased following TIM treatment, whereas the protein expression of NOXA was upregulated. Addition of the MCL-1 stabilization agent $\mathrm{PGE}_{1}$ significantly antagonized the anti-proliferative effects of TIM on U2OS cells, indicating that the NOXA/MCL-1 axis was involved in TIM-induced apoptosis in U2OS cells (Fig. 4A and B). Furthermore, TIM treatment triggered ER stress, as evidenced by the upregulation of the gene and protein expression levels of ER stress markers IRE1, ATF6 and GRP78 (Fig. 4C and D).

TIM inhibits tumor growth in a xenograft mouse model. Tumor-bearing mice were treated with TIM at a concentration of 1 or $2 \mathrm{mg} / \mathrm{kg}$ for 24 days. It was observed that TIM treatment at $2 \mathrm{mg} / \mathrm{kg}$ significantly inhibited the U2OS tumor growth, as indicated by the reduction in tumor volume and weight, which showed stronger anti-tumor activities than the positive control drug, doxorubicin (Fig. 5A and B). Furthermore, TIM 
A

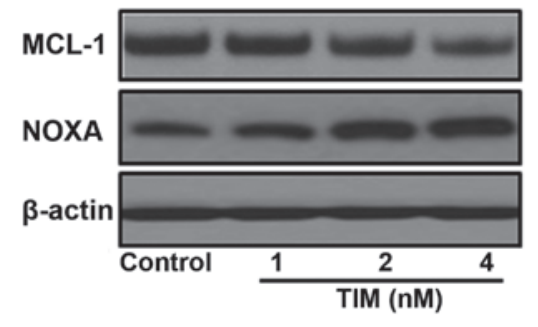

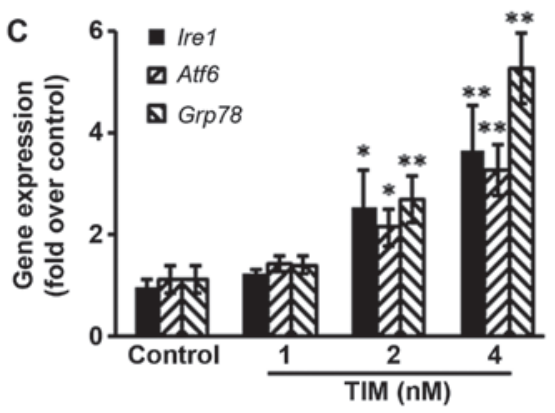
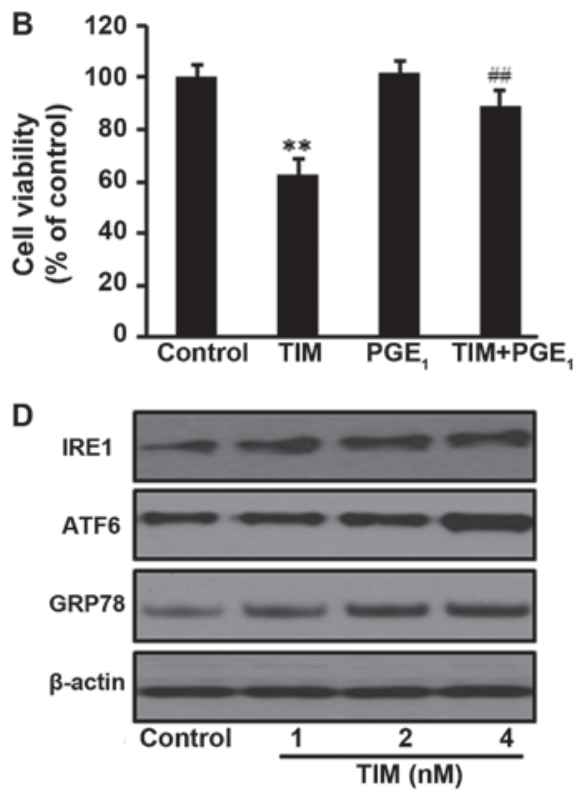

Figure 4. TIM induced apoptosis through the NOXA/MCL-1 axis and triggering ER stress. U2OS cells were treated with $4 \mathrm{nM}$ TIM for $48 \mathrm{~h}$. (A) TIM treatment decreased the protein expression of MCL-1, but increased the protein expression of NOXA. (B) PGE1 (100 nM) inhibited the anti-proliferative effects of TIM. (C) Gene and (D) protein expression levels of the ER stress-marker proteins IRE1, ATF6 and GRP78 were increased by TIM. Data are expressed as the mean \pm standard deviation $(\mathrm{n}=3)$. ${ }^{*} \mathrm{P}<0.05$ and ${ }^{* *} \mathrm{P}<0.01$, vs. control group; ${ }^{\# \#} \mathrm{P}<0.01$, vs. TIM alone. TIM (3R)-5,6,7-trihydroxy-3-isopropyl-3-methylisochroman-1-one; ER, endoplasmic reticulum; GRP78, glucose-regulated protein 78; IRE1, inositol-requiring enzyme 1; ATF6, activating transcription factor 6; PGE1, prostaglandin E1.

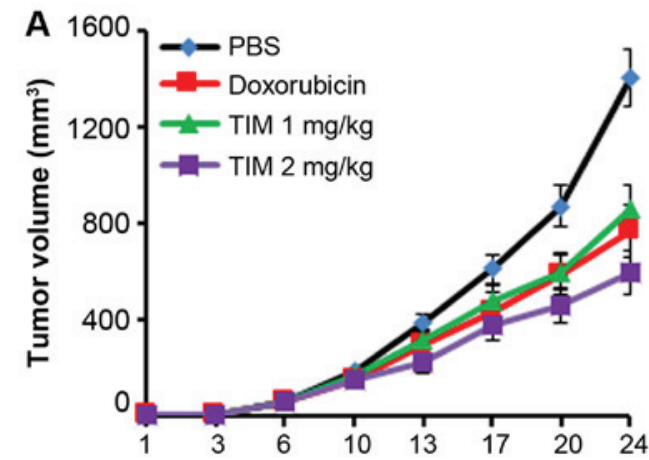

Days after tumor cell injection

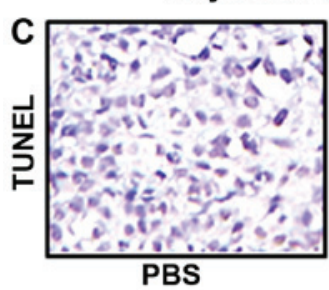

PBS

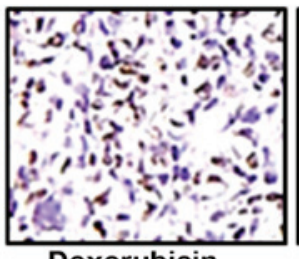

Doxorubicin
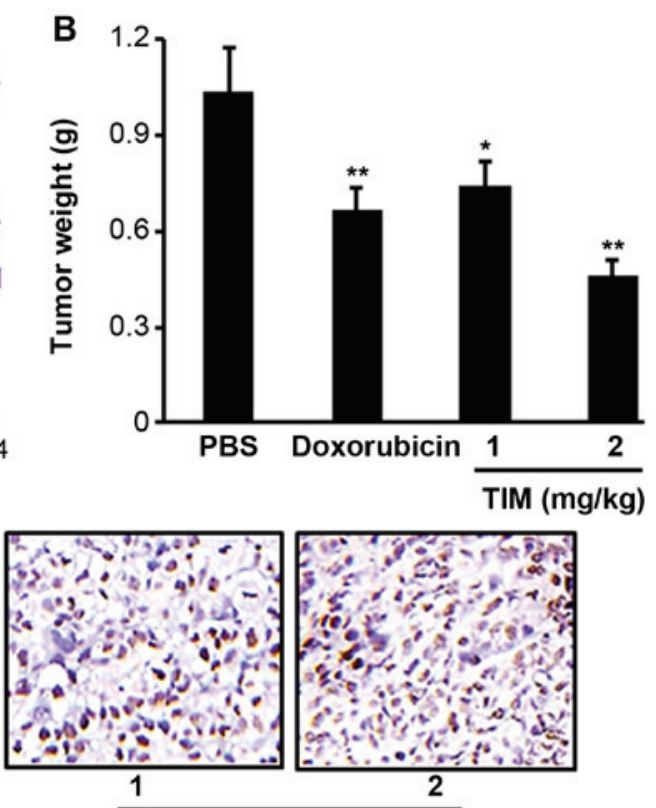

$\mathrm{TIM}(\mathrm{mg} / \mathrm{kg})$

Figure 5. TIM treatment inhibited osteosarcoma growth in nude mice. (A) Tumor volume was measured twice per week. (B) Tumor weight was recorded at the end of the experiment. (C) TIM treatment significantly increased the DNA fragmentation. Data are expressed as the mean \pm standard deviation $(\mathrm{n}=5)$. ${ }^{*} \mathrm{P}<0.05$ and ${ }^{* *} \mathrm{P}<0.01$, vs. PBS-treated control group. TIM, (3R)-5,6,7-trihydroxy-3-isopropyl-3-methylisochroman-1-one.

treatment was found to significantly increase the DNA fragmentation, as indicated by the TUNEL assay results (Fig. 5C).

\section{Discussion}

Current treatments for osteosarcoma consist of multi-agent chemotherapy, followed by complete surgical resection, radiation therapy and surveillance for lung metastasis. However, patients with metastasis at the time of diagnosis exhibit a poor response to conventional therapies due to high tumor malignancy and inadequate treatment efficacy $(17,18)$. Therefore, it is of utmost importance to search for effective novel agents and treatments. Herbal compounds are becoming an important source of medicinal products. In the present study, it 
was reported that TIM, a natural compound extracted from whole plants of Selaginella moellendorffii Hieron., inhibited osteosarcoma growth by inducing apoptosis.

The intrinsic mitochondrial pathway is a major signaling cascade for apoptosis. The role of mitochondria during the process of cell death regulation is crucial, and the decrease of MMP would induce the release of cytochrome $c$ from mitochondria to the nucleus, activating caspase-associated apoptotic proteins (19,20). Caspase-3 and caspase-9 activate endonucleases to cleave nuclear DNA and ultimately lead to DNA fragmentation (21). The current study results revealed that TIM decreased the MMP, while it increased the cytochrome $c$ release, DNA fragmentation, and activities of caspase- 3 and caspase- 9 . Furthermore, apoptosis significantly increased following TIM treatment. It was observed that the expression of the anti-apoptotic protein B-cell lymphoma 2 (Bcl-2) decreased, whereas the pro-apoptotic protein Bcl-2-associated X protein (Bax) and caspase expression levels increased upon TIM treatment.

Members of the $\mathrm{Bcl}-2$ family serve key roles in the regulation of apoptotic processes in numerous cancer cells (9). NOXA, a crucial pro-apoptotic protein in the Bcl-2 family, interacts with the anti-apoptotic Bcl-2 protein MCL-1, interfering with the polymerization of Bax and Bak to trigger apoptosis $(22,23)$. The NOXA/MCL-1 axis has been reported to be involved in apoptosis induced by chemotherapeutics in a number of tumor models $(9,23)$. In the present study, the expression of MCL-1 decreased upon TIM treatment, while the pro-apoptotic protein NOXA was upregulated. Furthermore, the addition of $\mathrm{PGE}_{1}$, an MCL-1 stabilization agent (24), antagonized the anti-proliferative effects of TIM on U2OS cells. These results indicated that TIM induced intrinsic mitochondrial apoptosis through the NOXA/MCL-1 axis in osteosarcoma cells.

Various chemotherapeutics activate ER response while inducing apoptosis in cancer cells (25). As a central regulator of ER stress to control the activation of transmembrane ER stress sensors (IRE1 and ATF6), GRP78 has been established by a large number of studies as a marker of ER stress (26). As signaling proteins in ER stress, IRE1 activates the apoptosis-signaling kinase-1, which further activates downstream of stress kinases to promote apoptosis, and ATF6 translocates as a transcriptional factor to the Golgi compartment where it is cleaved upon ER stress (27). The current study results demonstrated that ER stress marker proteins were upregulated upon TIM treatment, suggesting that TIM triggered ER stress in osteosarcoma cells.

However, in the current study, only one osteosarcoma cell line was analyzed in detail, which poses a limitation. Thus, further studies are required to validate the effects of TIM on osteosarcoma.

In conclusion, the present study demonstrated that TIM, isolated from whole plants of Selaginella moellendorffii Hieron., inhibited osteosarcoma growth, by inducing apoptosis via the NOXA/MCL-1 axis and ER stress. The results provide novel insight that may assist in the development of TIM as a therapeutic agent against osteosarcoma.

\section{Acknowledgements}

The authors thank Professor Yi He from Department of Pharmaceutical Science, Hangzhou Vocational and Technical College for providing TIM in the current study.

\section{Funding}

No funding received.

\section{Availability of data and materials}

The data are available from the corresponding author upon reasonable request.

\section{Authors' contributions}

LJL design and conceived in the current study. MZS and FLZ performed the studies and analyzed the data. LJL, MZS and FLZ prepared the manuscript.

\section{Ethics approval and consent to participate}

All animal experiments were performed in compliance with the Chinese legislation on the use and care of laboratory animals, and the study was approved by the Ethical Committee on Animal Care and Use of Qingdao University Medical College (Yantai, China).

\section{Patient consent for publication}

Not applicable.

\section{Competing interests}

The authors declare that they have no competing interests.

\section{References}

1. Lussier DM, Johnson JL, Hingorani P and Blattman JN: Combination immunotherapy with $\alpha$-CTLA-4 and $\alpha$-PD-L1 antibody blockade prevents immune escape and leads to complete control of metastatic osteosarcoma. J Immunother Cancer 3: 21, 2015.

2. Li Y, Du W, Han J and Ge J: LAMP3 promotes the invasion of osteosarcoma cells via SPP1 signaling. Mol Med Rep 16: 5947-5953, 2017.

3. Carrle D and Bielack S: Osteosarcoma lung metastases detection and principles of multimodal therapy. Cancer Treat Res 152: 165-184, 2009.

4. Harting MT and Blakely ML: Management of osteosarcoma pulmonary metastases. Semin Pediatr Surg 15: 25-29, 2006.

5. Strauss SJ,Ng T, Mendoza-Naranjo A, Whelan J and Sorensen PH: Understanding micrometastatic disease and Anoikis resistance in ewing family of tumors and osteosarcoma. Oncologist 15: 627-635, 2010.

6. Feng Y, Yang Y, Fan C, Di S, Hu W, Jiang S, Li T, Ma Z, Chao D, Feng X, et al: Pterostilbene inhibits the growth of human esophageal cancer cells by regulating endoplasmic reticulum stress. Cell Physiol Biochem 38: 1226-1244, 2016.

7. Uemura $\mathrm{N}$ and Kondo T: Current advances in esophageal cancer proteomics. Biochim Biophys Acta 1854: 687-695, 2015.

8. Yang Q, Mas A, Diamond MP and Al-Hendy A: The mechanism and function of epigenetics in uterine leiomyoma development. Reprod Sci 23: 163-175, 2016.

9. Zhao X, Kong F, Wang L and Zhang H: c-FLIP and the NOXA/Mcl-1 axis participate in the synergistic effect of pemetrexed plus cisplatin in human choroidal melanoma cells. PLoS One 12: e0184135, 2017.

10. Wang H, Yang Y, Chen H, Dan J, Cheng J, Guo S, Sun X, Wang W, Ai Y, Li S, et al: The predominant pathway of apoptosis in THP-1 macrophage-derived foam cells induced by 5 -aminolevulinic acid-mediated sonodynamic therapy is the mitochondria-caspase pathway despite the participation of endoplasmic reticulum stress. Cell Physiol Biochem 33: 1789-1801, 2014. 
11. Beck D, Niessner H, Smalley KS, Flaherty K, Paraiso KH Busch C, Sinnberg T, Vasseur S, Iovanna JL, Drieben S, et al: Vemurafenib potently induces endoplasmic reticulum stress-mediated apoptosis in BRAFV600E melanoma cells. Sci Signal 6: ra7, 2013.

12. Lee JH, Kwon EJ and Kim DH: Calumenin has a role in the alleviation of ER stress in neonatal rat cardiomyocytes. Biochem Biophys Res Commun 439: 327-332, 2013.

13. Chow SE, Kao CH, Liu YT, Cheng ML, Yang YW, Huang YK Hsu CC and Wang JS: Resveratrol induced ER expansion and ER caspase-mediated apoptosis in human nasopharyngeal carcinoma cells. Apoptosis 19: 527-541, 2014.

14. Zhou XF, Tong GT, Wang XW and He Y: Anti-proliferative constituents from Selaginella moellendorffii. Nat Prod Commun 11: 623-626, 2016.

15. Livak KJ and Schmittgen TD: Analysis of relative gene expression data using real-time quantitative PCR and the 2(-Delta Delta C(T)) method. Methods 25: 402-408, 2001

16. Hauck P, Chao BH, Litz J and Krystal GW: Alterations in the Noxa/Mcl-1 axis determine sensitivity of small cell lung cancer to the BH3 mimetic ABT-737. Mol Cancer Ther 8: 883-892, 2009.

17. Ferlay J, Soerjomataram I, Dikshit R, Eser S, Mathers C, Rebelo M, Parkin DM, Forman D and Bray F: Cancer incidence and mortality worldwide: Sources, methods and major patterns in GLOBOCAN 2012. Int J Cancer 136: E359-E386, 2015.

18. Saraf AJ, Fenger JM and Roberts RD: Osteosarcoma: Accelerating progress makes for a hopeful future. Front Oncol 8: 4, 2018.

19. Wang Z, Lu W, Li Y and Tang B: Alpinetin promotes Bax translocation, induces apoptosis through the mitochondrial pathway and arrests human gastric cancer cells at the G2/M phase. Mol Med Rep 7: 915-920, 2013.
20. Xue S, Chen YX, Qin SK, Yang AZ, Wang L, Xu HJ and Geng HY: Raltitrexed induces mitochondrial-mediated apoptosis in SGC7901 human gastric cancer cells. Mol Med Rep 10: 1927-1934, 2014.

21. Xin BR, Liu JF, Kang J and Chan WP: (2R, 3S)-pinobanksin3-cinnamate, a new flavonone from seeds of Alpinia galanga willd., presents in vitro neuroprotective effects. Mol Cell Toxicol 10: 165-172, 2014.

22. Zhao X, Liu X and Su L: Parthenolide induces apoptosis via TNFRSF10B and PMAIP1 pathways in human lung cancer cells. J Exp Clin Cancer Res 33: 3, 2014.

23. Liu X, Lv Z, Zou J, Liu X, Ma J, Wang J, Sa N, Jing P and $\mathrm{Xu}$ W: Afatinib down-regulates MCL-1 expression through the PERK-eIF2 $\alpha$-ATF4 axis and leads to apoptosis in head and neck squamous cell carcinoma. Am J Cancer Res 6: 1708-1719, 2016.

24. Kato T, Kutsuna H, Oshitani N and Kitagawa S: Cyclic AMP delays neutrophil apoptosis via stabilization of Mcl-1. FEBS Lett 580: 4582-4586, 2006.

25. Qin L, Wang Z, Tao L and Wang Y: ER stress negatively regulates AKT/TSC/mTOR pathway to enhance autophagy. Autophagy 6: 239-247, 2010.

26. Lee AS: The ER chaperone and signaling regulator GRP78/BiP as a monitor of endoplasmic reticulum stress. Methods 35: 373-381, 2005.

27. Sano R and Reed JC: ER stress-induced cell death mechanisms. Biochim Biophys Acta 1833: 3460-3470, 2013.

(i) (9) This work is licensed under a Creative Commons Attribution-NonCommercial-NoDerivatives 4.0 International (CC BY-NC-ND 4.0) License. 